\title{
Magnetic Field Dependence of Critical Attenuation
}

\section{in Ferromagnet}

\author{
A. PAWLAK* \\ Faculty of Physics, Adam Mickiewicz University, Umultowska 85, 61-614 Poznań, Poland
}

\begin{abstract}
The influence of magnetic field on the sound attenuation is investigated in ferromagnets. With the aid of the Nelson method we derive frequency-, magnetic field- and temperature-dependent expression for the sound attenuation coefficient near a critical point in the Ising-type system. The shift of the ultrasonic attenuation peak under the influence of magnetic field towards higher temperatures is discussed.
\end{abstract}

PACS: 05.70.Jk, 62.65. $+\mathrm{k}$

\section{Introduction}

Many aspects of sound attenuation above and below critical point have been discussed in the literature [1,2]. However, little attention has been paid so far to critical attenuation in the presence of the magnetic field. A notable exception are the mean-field theory results [3-5]. The aim of this paper is to provide a dynamical matching formalism of the renormalization group which allows us to calculate the ultrasonic attenuation coefficient $\alpha(t, \omega, H)$ by means of the $\epsilon$ expansion not only in the asymptotic region $H=0$ but also in the whole magnetic-field range. We implement the method used in the calculation of the dynamic susceptibility in the ordered phase [6] for the evaluation of the sound attenuation coefficient in an external magnetic field but use a modified matching condition suitable for $H \neq 0$.

One of the best method for computation of the scaling functions is the method introduced by Nelson [7] for computation of the static correlation function above the critical temperature. It was a generalization of the renormalization group technique developed by Nelson and Rudnick [8] used to obtain the equation of state to first order in $\epsilon=4-d$ as well as the scaling functions for the susceptibility, specific heat and etc. both in the ordered and disordered phase. Later this method was generalized by Achiam and Kosterlitz [9] to calculate the static momentum-dependent correlation function for arbitrary temperature and magnetic field. Dengler et al. [10] were first who generalized this method into dynamic correlation function in the disordered phase. In a recent paper Pawlak and Erdem [6] extended these results by obtaining (to first order in $\epsilon$ ) the expressions for the dynamic susceptibility and correlation function for nonconserved Ising order parameter both above and below the critical temperature in zero magnetic field. Generalization of this method to nonzero fields will be used for calculation of the ultrasonic attenuation.
It is well known that in the absence of the magnetic field, the critical behaviour of the sound attenuation coefficient is characterized by the scaling relations $[2,11,12]$. $\alpha(t, \omega) \propto \omega^{2}|t|^{-\rho_{\mathrm{s}}} f_{ \pm}\left(y_{ \pm}\right)$, where $f_{ \pm}$are the scaling functions above and below $T_{\mathrm{C}}, y_{ \pm}=\omega \tau_{ \pm}^{0}|t|^{-z \nu}$ the reduced frequencies whereas $\omega$ is the ultrasonic frequency; $\tau_{ \pm}^{0}$ are bare critical relaxation times for the order parameter fluctuations (above and below $T_{\mathrm{C}}$ ) and $\rho_{\mathrm{s}}$ is the critical sound attenuation exponent. In the magnets that have also metallic properties large sound attenuation critical exponents are usually measured $[1,2]$ and the so-called Murata-Iro-Schwabl regime is expected [2, 12-14], where $\rho_{\mathrm{s}}=z \nu+\alpha$ can be expressed by specific-heat $(\alpha)$ and correlation length $\left(\nu=1 / \lambda_{\mathrm{t}}\right)$ exponents and the dynamic critical exponent $z$. In the absence of the magnetic field the sound attenuation maximum as well as the sound velocity minimum occur slightly below the transition temperature, i.e. at the temperature at which the reduced frequency $y_{-} \approx 1$. The peak in the sound attenuation coefficient below the transition temperature is due to the static polarization $M=\langle S(x)\rangle$ of the order parameter and it is the analogue of the Landau-Khalatnikov (LK) sound damping [3]. It is the only term which contributes to $\alpha(t, \omega)$ in the mean-field theory.

The sound attenuation coefficient and velocity are strongly affected by an external magnetic field $[1,2,5]$. If the magnetic field is applied along the easy axis of a ferromagnet, it was observed $[1,15]$ that the ultrasonic attenuation peak is shifted towards higher temperatures and can be located even at the temperatures much higher than $T_{\mathrm{C}}$. Our theory explains the magnetic field dependence of ultrasonic attenuation. The shift of the attenuation peak is due to a competition between the fluctuation term (which is the only one present in zero magnetic field above $T_{\mathrm{C}}$ ) and the Landau-Khalatnikov term, which appears whenever a magnetization takes a non-zero value (so also for $T>T_{\mathrm{C}}, H \neq 0$ ). Only the Murata-IroSchwabl regime is considered in this paper.

* e-mail: pawlak@amu.edu.pl 


\section{Model and the solutions of the recursion relations}

We consider Ising-like $(n=1)$ continuous order parameter $S$ with purely dissipative relaxational dynamics

$$
\dot{S}_{\boldsymbol{k}}=-\Gamma \frac{\delta \mathcal{H}}{\delta S_{-k}}+\zeta_{\boldsymbol{k}}
$$

and longitudinal sound mode $Q$ obeying the equation

$$
\ddot{Q}_{\boldsymbol{k}}=-\frac{\delta \mathcal{H}}{\delta Q_{-\boldsymbol{k}}}-\theta k^{2} \dot{Q}_{\boldsymbol{k}}+\eta_{\boldsymbol{k}},
$$

with the Ginzburg-Landau Hamiltonian

$$
\begin{aligned}
\mathcal{H} & =\frac{1}{2} \sum_{\boldsymbol{k}}\left(r+k^{2}\right)\left|S_{\boldsymbol{k}}\right|^{2}+k^{2} c_{0 L}^{2}\left|Q_{\boldsymbol{k}}\right|^{2} \\
& +\sum_{\boldsymbol{k}, \boldsymbol{k}_{1}} g_{0} k Q_{\boldsymbol{k}} S_{\boldsymbol{k}_{1}} S_{-\boldsymbol{k}-\boldsymbol{k}_{1}} \\
& +\frac{u}{V} \sum_{\boldsymbol{k}, \boldsymbol{k}_{1}, \boldsymbol{k}_{2}} S_{\boldsymbol{k}} S_{\boldsymbol{k}_{1}} S_{\boldsymbol{k}_{2}} S_{-\boldsymbol{k}-\boldsymbol{k}_{1}-\boldsymbol{k}_{2}}
\end{aligned}
$$

and the Gaussian white noises obeying the Einstein relations

$$
\begin{aligned}
& \left\langle\zeta(\boldsymbol{k}, t) \zeta\left(\boldsymbol{k}^{\prime}, t^{\prime}\right)\right\rangle=2 \Gamma \delta\left(t-t^{\prime}\right) \delta_{\boldsymbol{k},-\boldsymbol{k}^{\prime}}, \\
& \left\langle\eta(\boldsymbol{k}, t) \eta\left(\boldsymbol{k}^{\prime}, t^{\prime}\right)\right\rangle=2 \theta k^{2} \delta\left(t-t^{\prime}\right) \delta_{\boldsymbol{k},-\boldsymbol{k}^{\prime}} .
\end{aligned}
$$

The scaling relation for the ultrasonic attenuation coefficient at the $l$-th stage of iteration is

$$
\frac{\alpha(\omega, \tau, u, M)}{\omega^{2}}=\mathrm{e}^{\rho_{\mathrm{s}} l / \nu} \frac{\alpha\left(\omega \mathrm{e}^{z l}, \tau(l), u(l), M(l)\right)}{\left(\omega \mathrm{e}^{z l}\right)^{2}},
$$

where $\tau(l)=\tau \mathrm{e}^{2 l} / Q(l)^{1 / 3}, u(l)=u \mathrm{e}^{\epsilon l} / Q(l)$ and $M(l)=$ $M \mathrm{e}^{\lambda_{m} l^{*}}$ are the renormalized temperature, coupling constant, and magnetization at the $l$-th stage of the renormalization group with $\tau=r+6 K_{4} u \propto T-T_{\mathrm{c}}, \lambda_{m}=$ $\beta / \nu=1-\frac{\epsilon}{2}+\mathrm{O}\left(\epsilon^{2}\right), K_{4}=1 / 8 \pi^{2}$, and $Q(l)=1+$ $36 K_{4} u\left(\mathrm{e}^{\epsilon l}-1\right) / \epsilon[8]$.

The technique developed by Nelson consists in mapping the Hamiltonian out of critical regime into noncritical region with small correlation length, where the usual perturbation expansion can be employed. Subsequently, the quantity calculated in the noncritical regime is related by a renormalization group ( $R G$ ) transformation to the corresponding quantity in the critical region. The original matching condition $\tau\left(l^{*}\right)+q^{2} \mathrm{e}^{2 l^{*}}=1$ introduced by Nelson [7] in order to evaluate the two-point correlation function outside the critical region is replaced here by

$$
\left[(\omega / \Gamma) \mathrm{e}^{z l^{*}}\right]^{4 / z}+\left[\tau\left(l^{*}\right)+12 u_{\mathrm{c}} M\left(l^{*}\right)^{2}\right]^{2}=1,
$$

where we have fixed $u$ at its fixed point value $u_{\mathrm{c}}=$ $\epsilon / 36 K_{4}$ and used the fact that in the ultrasonic experiments the wavelength is much longer than the correlation length so we may take the wavevector $q=0$. The perturbation expansion for $\alpha\left(\omega \mathrm{e}^{z l^{*}}, \tau\left(l^{*}\right), M\left(l^{*}\right)\right)$ is easily obtained

$$
\begin{aligned}
& \frac{\alpha\left(\omega \mathrm{e}^{z l^{*}}, \tau\left(l^{*}\right), M\left(l^{*}\right)\right)}{\left(\omega \mathrm{e}^{z l^{*}}\right)^{2}}=\frac{A}{\omega \mathrm{e}^{z l^{*}}} \operatorname{Im}\left\{\frac{M\left(l^{*}\right)^{2}}{E\left(l^{*}\right)} G\left(l^{*}\right)\right. \\
& +\left[\frac{24 u_{\mathrm{c}} M\left(l^{*}\right)^{2}}{E\left(l^{*}\right)}\right]^{2} G\left(l^{*}\right)^{2} \Pi\left(l^{*}\right) \\
& \left.-48 u_{\mathrm{c}} \frac{M\left(l^{*}\right)^{2}}{E\left(l^{*}\right)} G\left(l^{*}\right) \Pi\left(l^{*}\right)+\Pi\left(l^{*}\right)\right\},
\end{aligned}
$$

where

$$
\begin{aligned}
& G\left(l^{*}\right)=\left[1-\mathrm{i} w\left(l^{*}\right)\right]^{-1}, \quad w\left(l^{*}\right)=\omega \mathrm{e}^{z l^{*}} / E\left(l^{*}\right) \Gamma, \\
& \Pi\left(l^{*}\right)=-\frac{K_{4}}{4}\left\{\ln E\left(l^{*}\right)+\ln \left[1-\frac{\mathrm{i} w\left(l^{*}\right)}{2}\right]\right. \\
& \left.+\frac{2 \mathrm{i}}{w\left(l^{*}\right)} \ln \left[1-\frac{\mathrm{i} w\left(l^{*}\right)}{2}\right]\right\}
\end{aligned}
$$

and $A$ is a constant. It is a standard procedure $[7,9,10]$ to include in the static self-energy of the spin propagators the Hartree bubble given by an integral over the exact correlation function. Since such diagram is independent of the external frequency the matching condition (7) does not prevent it from being infrared singular at $\tau=0$. It can be however identified with the energy at the $l$-th stage and its singularity can be exponentiated by a similar procedure but with $\omega$-independent condition

$$
\tau\left(l^{*}\right) \mathrm{e}^{\lambda_{\mathrm{t}} L}+12 u_{\mathrm{c}} M\left(l^{*}\right)^{2} \mathrm{e}^{2 \lambda_{m} L}=1,
$$

which leads to following expression for $E\left(l^{*}\right)[6,9]$ :

$$
\begin{aligned}
& E\left(l^{*}\right)=2 \tau\left(l^{*}\right)-\tau\left(l^{*}\right) \mathrm{e}^{\alpha L / \nu}+M\left(l^{*}\right)^{2} \mathrm{e}^{\left(2 \lambda_{m}+\lambda_{\mathrm{t}}-d\right) L} \\
& \quad+\mathrm{O}\left(\epsilon^{2}\right),
\end{aligned}
$$

where $\mathrm{e}^{L}$ is expressed by $\tau\left(l^{*}\right)$ and $M\left(l^{*}\right)$ from Eq. (9).

\section{Magnetic field dependence of the ultrasonic attenuation}

The solutions of the matching conditions (7) and (9) are homogeneous functions of the form

$$
\begin{aligned}
& \mathrm{e}^{l^{*}}=m^{-1 / \lambda_{m}} F(s, x), \\
& \mathrm{e}^{L}=m\left(l^{*}\right)^{-1 / \lambda_{m}} \Phi(s),
\end{aligned}
$$

where $s=2 \tau m^{-1 / \beta}, x=2 \tau(\omega / \Gamma)^{-1 / z \nu}$. We have rescaled the magnetization by defining $m^{2}=8 u_{\mathrm{c}} M^{2}$ and then Eqs. (7) and (9) can be written as

$$
\begin{aligned}
& 4\left(\frac{s}{x}\right)^{2 z \nu} F(s, x)^{2 z}+\left[s F(s, x)^{\lambda_{\mathrm{t}}}+3 F(s, x)^{2 \lambda_{m}}\right]^{2}=4, \\
& s \Phi(s)^{\lambda_{\mathrm{t}}}+3 \Phi(s)^{2 \lambda_{m}}=2 .
\end{aligned}
$$

In order to obtain the magnetic field dependence of $\alpha(\omega, \tau, M)$ we need the equation of state which is the solution of (14) and

$$
h m^{-\delta}=\frac{s \Phi(s)^{\lambda_{\mathrm{t}}+2 \lambda_{m}-d}+\Phi(s)^{4 \lambda_{m}-d}}{\Phi(0)^{4 \lambda_{m}-d}} \equiv f(s),
$$

where $h$ is a reduced magnetic field proportional to the 
module of the physical variable. In the critical point $\tau=0(s=0)$ we have $f(0)=1$ and on the coexistence curve $(\tau<0) s=-1$. We introduce now instead of $s$ a new variable $v=\tau h^{-1 /(\gamma+\beta)}$ which is related to $s$ via

$$
v=s f(s)^{-1 /(\gamma+\beta)},
$$

where $\gamma$ and $\delta$ are the critical exponents. Solving Eq. (16) for $s(v)$ we obtain $m(\tau, h)=\left(\frac{2 \tau}{s(v)}\right)^{\beta} \equiv|\tau|^{\beta} \hat{m}(v)$. The function $s(v)$ is shown in Fig. 1. Substitution of these results into Eqs. (6) and (8) gives our final expression for the sound attenuation coefficient

$$
\alpha(\omega, \tau, h)=A \omega^{2}|\tau|^{-\rho_{\mathrm{s}}} \hat{g}_{1}(v, x),
$$

where $\hat{g}_{1}$ is a scaling function. Defining a proper reduced frequency $y$ in terms of $x$ and $v$ it is possible to change variables to obtain

$$
\alpha(\omega, \tau, h)=A \omega^{2}|\tau|^{-\rho_{\mathrm{s}}} \hat{g}_{2}(v, y),
$$

where $\hat{g}_{2}(v, y)$ is another scaling function.

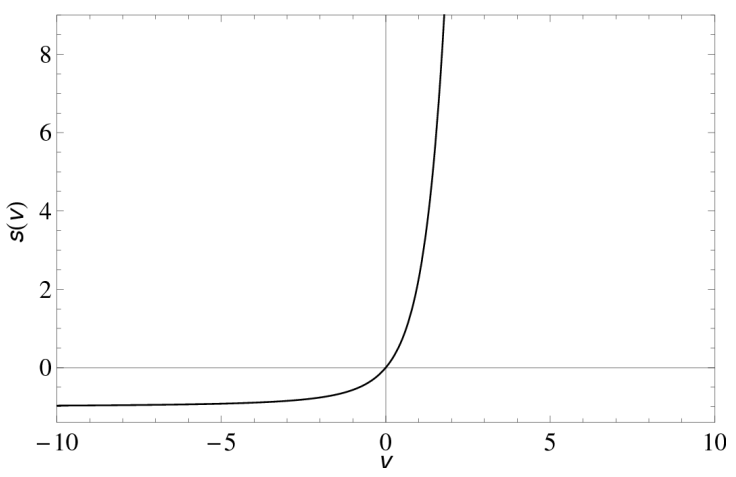

Fig. 1. The variable $s$ as a function of the field variable $v$.

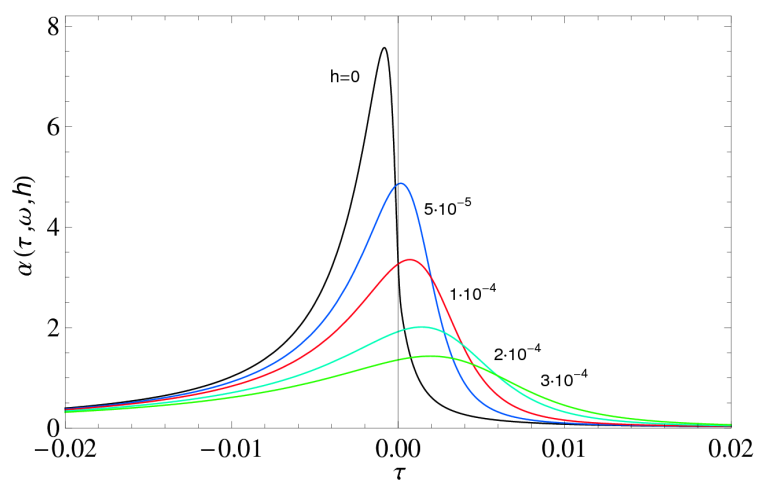

Fig. 2. The sound attenuation coefficient calculated to $\mathrm{O}(\epsilon)$ as a function of the reduced temperature. The values of reduced magnetic field are given for each curve.
We have also analyzed the position of the maximum in the sound attenuation coefficient as a function of the external magnetic field. Figure 2 shows that it is moved towards the higher temperatures as the field increases. For the temperatures far from the critical point the sound attenuation coefficient decreases with increasing field below $T_{\mathrm{C}}$ and increases above $T_{\mathrm{C}}$. Such behaviour has been reported in the experiments of Komatsubara et al. [15] for a single crystal MnP. It is connected with the fact that the dominant contribution to $\alpha(\omega, \tau, h)$ comes from the first term (LK term) in Eq. (8) which not only decreases with the increasing field but also "moves" towards the higher temperatures. The last contribution in Eq. (8) (fluctuation term) is an order of magnitude smaller than the LK term but it decreases with $h$ much slower than the first contribution. Our analysis shows that maximum of the fluctuation term also moves towards the higher temperatures. The height of the ultrasonic attenuation maximum decreases as $h^{-z \nu /(\beta+\gamma)}$ for high magnetic fields.

\section{References}

[1] B. Lúthi, Physical Acoustics in the Solid State, Springer, Berlin 2005.

[2] A. Pawlak, in: Horizons in World Physics, Vol. 268, Eds. L. Pedroza, M. Everett, Nova Science Publishers, Hauppauge (NY) 2009, p. 1.

[3] L.D. Landau, I.M. Khalatnikov, Dokl. Akad. Nauk SSSR 96, 469 (1954).

[4] R. Erdem, J. Magn. Magn. Mater 320, 2273 (2008).

[5] A. Pawlak, Acta Phys. Pol. A 115, 229 (2009).

[6] A. Pawlak, R. Erdem, Phys. Rev. B 83, 094415 (2011).

[7] D.R. Nelson, Phys. Rev. B 14, 1123 (1976).

[8] J. Rudnick, D.R. Nelson, Phys. Rev. B 13, 2208 (1976).

[9] Y. Achiam, J.M. Kosterlitz, J. Phys. C 10, 4559 (1977).

[10] R. Dengler, H. Iro, F. Schwabl, Phys. Lett. A 111, 121 (1985).

[11] R. Dengler, F. Schwabl, Z. Phys. B 69, 327 (1987).

[12] A. Pawlak, Eur. Phys. J. B 4, 179 (1998).

[13] H. Iro, F. Schwabl, Solid State Commun. 46, 205 (1983).

[14] K.K. Murata, Phys. Rev. B 13, 4015 (1976).

[15] T. Komatsubara, A. Ishizaki, S. Kusaka, E. Hirahara, Solid State Commun. 14, 741 (1974). 